\title{
Diagnostic Prediction for Social Anxiety Disorder via Multivariate Pattern Analysis of the Regional Homogeneity
}

\author{
Wenjing Zhang, ${ }^{1}$ Xun Yang, ${ }^{1,2}$ Su Lui, ${ }^{1,3}$ Yajing Meng, ${ }^{4}$ Li Yao, ${ }^{1}$ \\ Yuan Xiao, ${ }^{1}$ Wei Deng, ${ }^{4}$ Wei Zhang, ${ }^{4}$ and Qiyong Gong ${ }^{1}$ \\ ${ }^{1}$ Huaxi MR Research Center (HMRRC), Department of Radiology, West China Hospital, Sichuan University, Chengdu 610041, China \\ ${ }^{2}$ School of Sociality and Psychology, Southwest University for Nationalities, Chengdu 610041, China \\ ${ }^{3}$ Radiology Department of the Second Affiliated Hospital, Wenzhou Medical University, Wenzhou, Zhejiang 325027, China \\ ${ }^{4}$ Department of Psychiatry, West China Hospital, Sichuan University, Chengdu 610041, China
}

Correspondence should be addressed to Su Lui; lusuwcums@tom.com

Received 21 August 2014; Revised 29 October 2014; Accepted 21 November 2014

Academic Editor: Xi-Nian Zuo

Copyright (c) 2015 Wenjing Zhang et al. This is an open access article distributed under the Creative Commons Attribution License, which permits unrestricted use, distribution, and reproduction in any medium, provided the original work is properly cited.

\begin{abstract}
Although decades of efforts have been spent studying the pathogenesis of social anxiety disorder (SAD), there are still no objective biological markers that could be reliably used to identify individuals with SAD. Studies using multivariate pattern analysis have shown the potential value in clinically diagnosing psychiatric disorders with neuroimaging data. We therefore examined the diagnostic potential of regional homogeneity (ReHo) underlying neural correlates of SAD using support vector machine (SVM), which has never been studied. Forty SAD patients and pairwise matched healthy controls were recruited and scanned by restingstate fMRI. The ReHo was calculated as synchronization of fMRI signals of nearest neighboring 27 voxels. A linear SVM was then adopted and allowed the classification of the two groups with diagnostic accuracy of ReHo that was $76.25 \%$ (sensitivity $=70 \%$, and specificity $=82.5 \%, P \leq 0.001$ ). Regions showing different discriminating values between diagnostic groups were mainly located in default mode network, dorsal attention network, self-referential network, and sensory networks, while the left medial prefrontal cortex was identified with the highest weight. These results implicate that ReHo has good diagnostic potential in SAD, and thus may provide an initial step towards the possible use of whole brain local connectivity to inform the clinical evaluation.
\end{abstract}

\section{Introduction}

Previously termed social phobia, social anxiety disorder (SAD) was characterized by persistent fear of social or performance situations in which there is judgment or scrutiny by others [1]. As the most common anxiety disorder, SAD shows a high lifetime prevalence of $12 \%$ and a 12 -month prevalence of 7.1\% [2]. Early onset, delay, or avoidance in seeking treatment leads to significant social and occupational disability for individuals with SAD. Currently, according to the diagnostic criteria in DSM-IV (Diagnostic and Statistical Manual of Mental Disorders, Fourth Edition), the diagnosis of SAD is based on observed behaviors and examinations of psychiatric signs and symptoms. However, the use of such a symptombased approach would sometimes cause uncorrected diagnosis due to high rates of comorbidity with depressive conditions and substance abuse [3]. Therefore, it is necessary to establish other objective and reliable approaches or biological markers, which could be used to assist the diagnosis of SAD and improve the accuracy.

As a more objective approach, neuroimaging holds great promise for detecting abnormalities crucial to the pathophysiologic models of SAD. Resting-state functional magnetic resonance imaging (fMRI) studies have revealed that, relative to the healthy controls, SAD is associated with abnormal activation within amygdala and default model network (DMN) mainly involved [4-6]. The amygdala is thought to be important in the acquisition and expression of conditioned fear and also performs a protective role, allowing the organism to detect and avoid danger [4], whilst role of DMN may be relevant to social perception and self-referential processing which are underlying psychological symptoms and pathophysiological mechanisms in SAD [6,7]. These findings are valuable in helping us understand the functional changes 
TABLE 1: Demographic and clinical characteristics of SAD patients and healthy comparison subjects.

\begin{tabular}{|c|c|c|c|c|c|}
\hline \multirow{2}{*}{ Demographic and clinical characteristics } & \multicolumn{2}{|c|}{$\operatorname{SAD}(n=40)$} & \multicolumn{2}{|c|}{$\mathrm{HC}(n=40)$} & \multirow{2}{*}{$P$} \\
\hline & Mean & $\mathrm{SD}$ & Mean & $\mathrm{SD}$ & \\
\hline Age (years) & 25.95 & 6.48 & 24.80 & 3.35 & 0.323 \\
\hline Duration of illness (years) & 7.76 & 4.61 & & & \\
\hline Age of onset (years) & 18.15 & 6.70 & & & \\
\hline \multicolumn{6}{|l|}{ LSAS } \\
\hline Total & 65.42 & 21.23 & 33.80 & 22.01 & 0.000 \\
\hline Fear factor & 32.45 & 10.39 & 15.70 & 11.90 & 0.000 \\
\hline \multirow[t]{2}{*}{ Avoidance factor } & 32.47 & 11.91 & 17.85 & 11.34 & 0.000 \\
\hline & $N$ & $\%$ & $N$ & $\%$ & $P$ \\
\hline \multicolumn{6}{|l|}{ Gender } \\
\hline Female & 14 & 35 & 14 & 35 & 1.000 \\
\hline Male & 26 & 65 & 26 & 65 & \\
\hline
\end{tabular}

SAD, social anxiety disorder; HC, healthy controls; SD, standard deviation; LSAS, Liebowitz Social Anxiety Scale; $n / N$, number.

Significance levels were set at $P<0.05$.

which underlie clinical symptoms associated with SAD; however, the extrapolation of any potential biomarkers and the clinical translation of the results have been hindered by the group level inference of the data. For the imaging findings to be clinically useful, one must be able to make inferences at the individual rather than the group level.

Relative to traditional univariate methods, multivariate pattern analysis (MVPA) allows predictions individually and it takes the patterns of information that might be presented across multiple variables into account, therefore providing results that have higher translational applicability in clinical practice [8]. For fMRI data, MVPA involves whole brain pattern classification aimed at decoding information in the pattern of activation across all voxels that may distinguish between two classes at the individual level. The most commonly used MVPA for pattern recognition in neuroimaging literature is support vector machine (SVM) [9]: an algorithm uses a well-defined dataset to create decision function or "hyperplane" which can best distinguish between categories (in current study, patients and controls), and then the produced decision function or "hyperplane" will be used to predict which predefined group a new observation belongs to. These two phases are systematically known as training and testing [9]. The overall accuracy of the SVM depends on its sensitivity (i.e., the proportion of patients identified as having the disease) and specificity (i.e., the proportion of controls identified as not having the disease). In recent years, SVM algorithm has been successfully applied to classify various neuropsychiatric disorders and achieved good diagnostic accuracy [10].

To date, there have been only two studies using MVPA in SAD but concentrating on task-based fMRI and regional grey matter volume [11], or functional connectivity [7]. As reflected by resting-state fMRI, functional connectivity can reveal the synchronization of remote brain regions, while, by contrast, regional homogeneity ( $\mathrm{ReHo}$ ) has been developed to measure the local synchronization of spontaneous fMRI signals by calculating similarity of dynamic fluctuations of voxels within a given cluster, revealing important information about local connectivity, and reflects the temporal synchrony of the regional fMRI BOLD signals $[12,13]$. Abnormal ReHo is assumed to be associated with aberrant changes in the temporal aspects of the spontaneous neural activity in the regional brain [14] and may be a sign of disrupted local functionality [15]. More importantly, ReHo can indicate some unexpected hemodynamic responses that model-driven methods may fail to discover in resting-state fMRI [13]. Although being successfully applied to various neuropsychiatric disorders [14, 16-20], the ReHo approach has been little investigated in SAD.

Thus, we particularly used SVM to examine ReHo maps in differentiating SAD patients from healthy controls, which has never been investigated. The purposes were to find out whether SVM would allow accurate discrimination between diagnostic groups and, if so, which brain regions or intrinsic brain networks would principally contribute to the discrimination.

\section{Materials and Methods}

2.1. Participants. Forty Structured Interview for the DSMIV (SCID) Patient Edition confirmed SAD patients and an equal number of healthy controls were recruited at the Mental Health Centre of West China Hospital (Table 1). The Ethics Committee of West China Hospital, Sichuan University, has offered approval to our study and all participants gave written informed consent to their participation. Diagnosis of SAD was determined by consensus of two experienced psychiatrists. Psychological ratings and clinical symptoms associated with SAD were evaluated with the Liebowitz Social Anxiety Scale (LSAS). Of the 40 patients, 12 had the antianxiety medication but they underwent at least two-week washing-out prior to the MR examination.

Healthy controls were recruited from the local area via poster advertisements and were screened using the SCIDNon-Patient Version to ascertain the lifetime absence of 
psychiatric and neurological illness. It was confirmed that they had no history of psychiatric illness among their firstdegree relatives. All subjects' demographic characteristics and clinical variables were obtained by 2 experienced clinical psychiatrists before MR examinations. Patients with SAD and control subjects were pairwise matched in age, gender, and handedness (Table 1). The following exclusion criteria applied to both groups: (1) the existence of a neurological disorder or other psychiatric disorders, (2) substance abuse, (3) pregnancy, or (4) major physical illness such as cardiovascular disease or hepatitis, as assessed by clinical evaluations and medical records. T1-weighted and T2-weighted images of brain were inspected by an experienced neuroradiologist, and no scanning artifacts and gross abnormalities were observed in any participants.

2.2. MRI Acquisition. The MRI examinations were performed on a whole-body 3.0 T MR scanner (Siemens Trio, Erlangen, Germany) with a 12-channel head coil. Earplugs were employed to protect the hearing while foam pads upon them were used to restrict head motion during the scanning. The resting-state fMRI sensitized to changes in BOLD signal levels was obtained with a gradient-echo planar imaging sequence $\left(\mathrm{TR} / \mathrm{TE}=2000 / 30 \mathrm{~ms}\right.$; flip angle $\left.=90^{\circ}\right)$. A field of view $(\mathrm{FOV})$ of $240 \times 240 \mathrm{~mm}^{2}$ was used with an acquisition matrix $=64 \times 64$, producing 30 continuous axial slices with thickness $=5.0 \mathrm{~mm}$ with no gap and voxel size $=3.75 \times 3.75 \times$ $5 \mathrm{~mm}^{3}$ in-plane resolution in each brain volume. Each functional run contained 205 volumes of which the first 5 were discarded to ensure steady-state longitudinal magnetization and subjects' adaptation to the environment. All participants were simply instructed to keep still with their eyes closed and remain awake but not to think of anything in particular. After the scanning, the volumes of all subjects were corrected for the temporal difference and head motion by setting the translational or rotational parameters at the threshold of $\pm 1.5 \mathrm{~mm}$ or $\pm 1.5^{\circ}$.

2.3. Imaging Preprocessing. The $\mathrm{AMRI}$ data was preprocessed using Data Processing Assistant for Resting-State fMRI (DPARSF, http://www.restfmri.net, version 2.1), implemented within the MATLAB toolbox, to calculate the ReHo maps. This software involves an integrated image process mainly including slice timing, realignment, and normalization to the Montreal Neurological Institute echo planar imaging template (each voxel was resampled to $3 \times 3 \times 3 \mathrm{~mm}^{3}$ ), removing linear trend and the ReHo calculation. Given the fact that ReHo shows the similarity or synchronization of fMRI signals of nearest neighboring voxels and Kendall's coefficient of concordance (KCC) is used for the measurement based on the regional homogeneity hypothesis [13], we defined 27 nearest neighboring voxels as a cluster and a KCC value was given to the voxel at the center of this cluster. The individual ReHo map was generated in a voxel-wise fashion, and all ReHo maps were smoothed with a Gaussian filter of $4 \mathrm{~mm}$ full-width half maximum (FWHM) kernel to manage the anatomical variability that was not compensated for by spatial normalization.
2.4. Comparison of Demographic Characteristics and Variables. The Statistical Package for the Social Sciences (SPSS, version 18.0) will be used for the comparison of demographic variables. Differences in age and LSAS scores between groups were analyzed using the two-sample $t$-tests, whereas gender ratio was compared with Chi-square test, with significance levels setting at $P<0.05$.

2.5. Multivariate Pattern Analysis and Support Vector Machine. SVM as implemented in the PROBID software package (http://www.brainmap.co.uk/probid.htm, version 1.04) was employed and a linear kernel SVM was adopted to classify the diagnostic groups based on their ReHo maps. The detailed description of the application of SVM in MRI data has been given $[8,21]$. In the context of supervised multivariate classification method as SVM [22], individual brain scans were treated as points located at high-dimensional space defined by the ReHo map in the preprocessed images. In this high-dimensional space, a linear decision boundary was defined by a "hyperplane" that separated the individual brain scans according to a class label (i.e., patients versus controls). The optimal hyperplane was computed based on the whole multivariate pattern of ReHo map across each image and could most accurately capture the relationship between each example and its respective label. The algorithm is initially trained on a subset of the data $\langle x, c\rangle$ to find a hyperplane that best separates the input space according to the class labels $c$ (patients versus controls), where $x$ represents the input data (i.e., ReHo map). The linear kernel SVM adopted could reduce the risk of overfitting the data and allow direct extraction of the weight vector as an image (i.e., the SVM discrimination map). Furthermore, the linear kernel matrix implicated in PROBID could be precomputed and supplied to the classifier, an approach which affords a substantial increase in computational efficiency and permits whole brain classification without requiring explicit dimensionality reduction [23]. A parameter $C$, which controls the tradeoff between having zero training errors and allowing misclassifications in the linear model, was fixed at $C=1$ for all cases (default value). A grey matter mask of $3 \times 3 \times$ $3 \mathrm{~mm}$ was used to constrain the search of significant group differences in voxels/features within grey matter in the comparison of ReHo maps.

Consistent with previous studies using SVM on SAD $[7,11]$, a "leave-one-out" cross validation was used, which means a single subject of each group would be excluded from the training and was later used to test the capability of the classifier learned from the remaining subjects, to reliably distinguish between categories (in our study, SAD or controls). Each subject pair would undergo this procedure to make the accuracy of the SVM fully estimated [8]. Statistical significance of the overall classification accuracy was determined by permutation testing $[24,25]$, a nonparametric test that involved repeating the classification procedure 1000 times with a different random permutation of the training group labels and counting the number of permutations achieving higher sensitivity and specificity than the true labels. Finally, to show the multivariate discriminating pattern of ReHo 


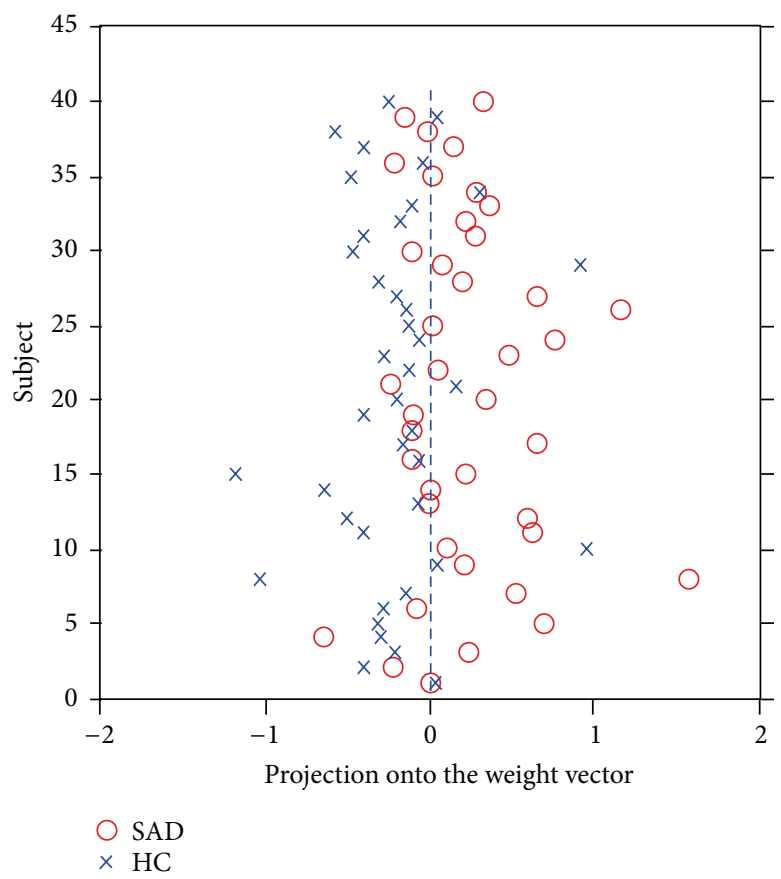

(a)

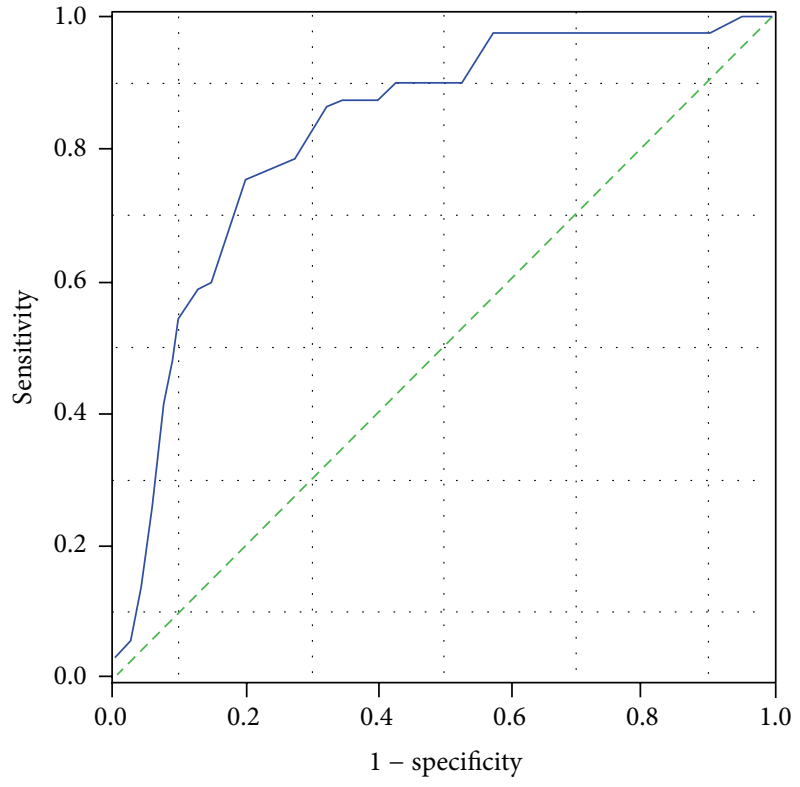

(b)

FIGURE 1: Classification plot (a) obtained from PROBID and receiver operating characteristic (ROC) curve and (b) obtained from SPSS for the discrimination between SAD patients and healthy controls using ReHo maps, yielding an accuracy of $76.25 \%$ (sensitivity $=70.0 \%$ and specificity $=82.5 \%, P \leq 0.001)$.

maps, a threshold would be set at $30 \%$ of the maximum weight vector value of the discrimination and voxels with greater value would be exhibited.

The test margin (the shortest distance from the optimal hyperplane), which could show the capability of the SVM with ReHo in classification of each subject, was calculated for all participants. Based on the label and test margin of each subject, the receiver operating characteristic (ROC) curve of the classification with ReHo maps was obtained with SPSS. To further explore whether the classification is driven by anxious symptoms and the extent if so, correlation analysis has been performed between the test margin and the level of symptom severity as determined by LSAS scores for all participants.

\section{Result}

3.1. Demographic and Clinical Characteristics. Demographic and clinical characteristics for all of participants are presented in Table 1. No significant differences were found in gender ratio and age between patients and healthy controls $(P>0.05)$. Compared to healthy control, SAD patients had significantly higher scores on the anxiety symptoms measured with LSAS total score and subscales $(P<0.05)$. All participants were right-handed. Twenty-eight patients were drug-naïve while the remaining 12 had taken different medication (5 paroxetine, 3 paroxetine with intermittent risperidone, alprazolam, and buspirone, resp., 3 sertraline, and 1 amitriptyline and doxepin) for 1 week to 5 years. The medicated patients had been drug-free for at least 2 weeks.
3.2. Multivariate Pattern Recognition. The classification of the two groups with overall diagnostic accuracy of ReHo maps was $76.25 \%$ (sensitivity $=70 \%$ and specificity $=82.5 \%$, $P \leq 0.001$ ) achieved by SVM (Figure 1). The set of regions showed different value between the diagnostic groups mainly located in frontal, temporal, and occipital regions (Figure 2, Table 2). In the discrimination map, a positive value means a relative higher weight in SAD (red scale) and helps in the identification of individuals with $\mathrm{SAD}$, with regions mainly located at right orbitofrontal gyrus (OFG), right middle frontal gyrus, right pars triangularis, right superior temporal gyrus (STG), left middle temporal gyrus (MTG), right postcentral gyrus (PCG), left inferior parietal lobe (IPL), and right precuneus, while a negative value means a relative higher weight in healthy controls and contributes to the identification of healthy subjects, locating in left medial prefrontal cortex (mPFC), bilateral middle frontal gyrus (MFG), right inferior occipital gyrus (IOG), and right cuneus (Figure 2).

3.3. Relationship between Test Margin and Severity of Symptom. Across all of the patients, the test margin was found not correlated to total LSAS scores, scores for fear factor, or scores for avoidance factor $(P>0.05)$.

\section{Discussion}

To the best of our knowledge, the current study is the first to examine the capability of SVM with ReHo in distinguishing patients with SAD from healthy subjects and involves 


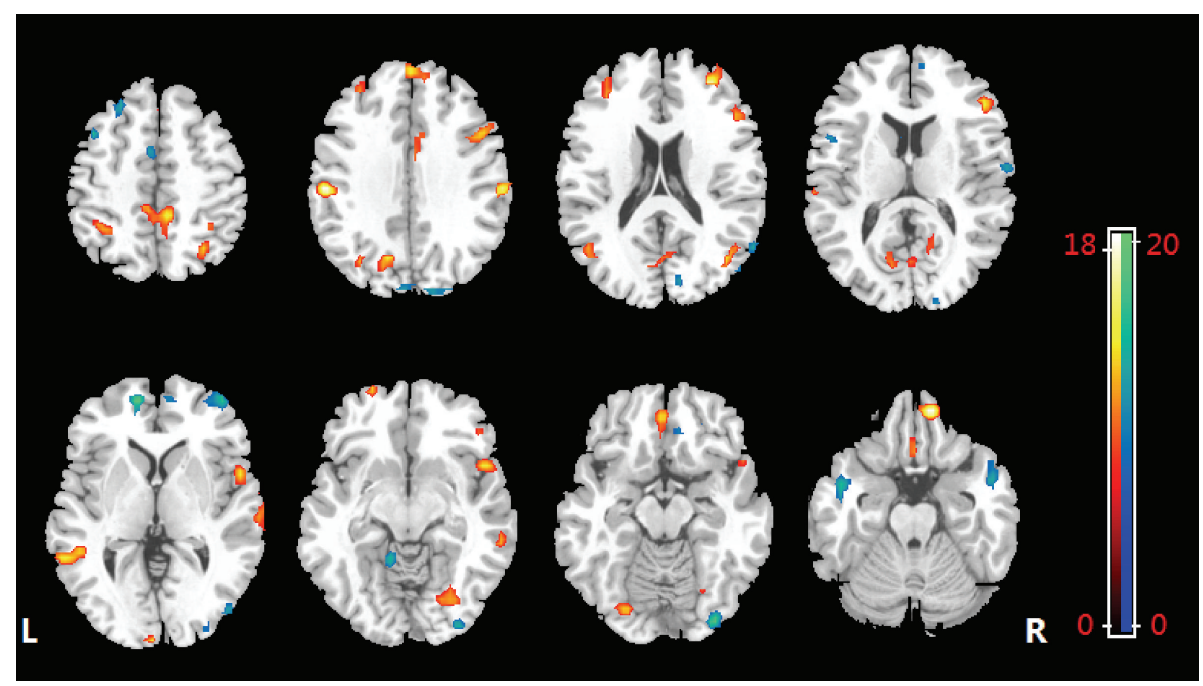

FIGURE 2: The discrimination maps for ReHo. Regions displayed were identified by setting the threshold to $\geq 30 \%$ of the weight vector scores. Warm color (positive value) indicated higher discriminated values in SAD than in healthy controls; cool color (negative weights) indicates higher values in healthy controls than in SAD.

the largest sample of SAD patients in employing MVPA approach. By identifying the intergroup differences in whole brain ReHo pattern with an overall classification accuracy of $76.25 \%$, the present study suggests local connectivity and synchronization extracted from fMRI BOLD signal could be a potential biomarker to identify SAD patients and demonstrates that multivariate analysis allows discrimination between individuals with SAD and healthy controls at relatively high level of accuracy. This pattern of results provides preliminary support to the development of SVM as a promising diagnostic tool in SAD to improve the diagnostic accuracy and minimize errors in detecting malingering where possible [26].

The discriminating pattern in the present study was attributable to widespread ReHo alterations, mainly involving DMN, dorsal attention network (DAN), self-referential network (SRN), and sensory networks. By contrast, the only one study using ReHo in SAD before found significantly decreased ReHo mainly in the DMN and central executive network (CEN) while it found increased ReHo in occipital regions and the right putamen in a relative small sample of SAD patients via mass-univariate analysis [27]. Relatively, our findings consistently revealed abnormalities within DMN but identified more regions, and other distributed regions across brain have also been showed with different local connectivity between SAD and control subjects. One explicable fact is that MVPA implicated in SVM takes the interregional correlation into account [8]. This multivariate nature of SVM rendered a high discriminative power for a given cluster deriving not only from differences in ReHo in that region between groups, but also from any intergroup differences in its functional correlations with other regions. Thus, the findings of the altered ReHo across brain should not be deemed as individual regions but as a spatially distributed pattern. Taken collectively, ReHo investigation gives insight into coherent local connectivity of a functional cluster and is necessary for further interpreting functional changes in SAD patients, whilst the combination with SVM identifies more distributed and subtle ReHo changes helpful in characterization at individual level therefore yielding results with great potential in clinical translation.

The consistent finding of altered local connectivity in relation to DMN emphasizes its critical role underlying the pathogenesis of SAD. The DMN is deemed as a higher-level cognitive network and consists of brain regions that typically activate during resting-state but deactivate during performance of goal-directed tasks [28], within which a set of regions connectively contributes to the social cognitive aspects. The precuneus, along with posterior cingulate cortex (PCC), is featured as the pivotal hub of DMN and related to perception of social cognition and self-related mental representations $[28,29]$. Patients with SAD showed a lower deactivation in regions comprising precuneus during task conditions [30] and abnormal functional connectivity in precuneus has also been suggested to be associated with the pathophysiological mechanism underlying SAD [31]. The mPFC is another hub in DMN and is identified with the highest weight. Activity in mPFC may reflect an interaction between cognitive processing and emotional state [32], especially for the anxiety-related emotion processing, in which mPFC has been considered of ongoing importance [33]. These data, along with our findings, supported the notion that $\mathrm{DMN}$ accounts for prominence in cognitive behavioral models of SAD.

DAN $[34,35]$ and SRN [36] are another two important networks with many regions found with alteration with ReHo in SAD patients. DAN is considered to mediate goal-directed (top-down) processing for stimuli selection and responses and involved in many higher-order cognitive tasks [34]. Particularly emotion regulation, the regulating of anxious feelings, has been emphasized in the cognitive models underlying SAD [37], and failure in emotion regulation has been considered another key feature of SAD [38]. In this term of view, we speculate that there is an important 


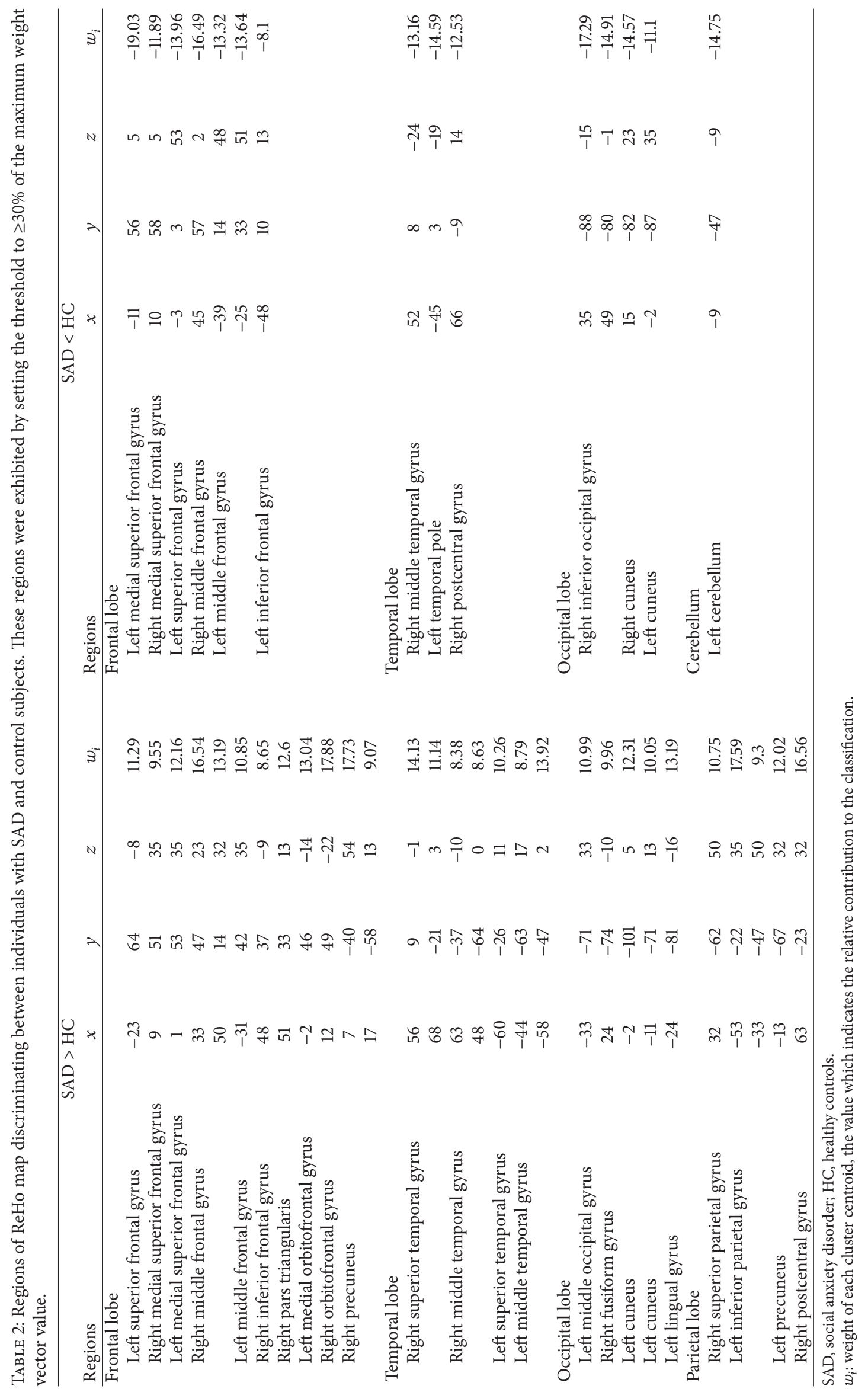


role of brain regions within DAN in emotion regulation, and abnormalities in these regions usually result in a high level of self-awareness, typically in SAD patients. While the SRN has exhibited peculiar physiological characteristics with increased neural activity during resting [28], OFC is a primary region observed with local coherence alteration. As involved in the engagement of interpersonal relationships, moral behavior, and social aggression [39-41], OFC with inappropriate function might strengthen the response to stressors or stimuli of fear conditions, resulting in severe impairments in social behavior [5]. Reduced orbitofrontal activation was observed in patients with SAD during public speaking [42] and anxiety-provoking tasks [43], implying a hypoactive OFC was associated with a failure of fear and anxiety inhibition.

There are still main regions implicated in visual network (VN), auditory network (AN), and somatomotor network (SMN). However, sensory networks could be regarded as the lower-order system of cognition. Within VN, abnormalities in IOG may be associated with the hypervigilance and hyperprosexia characteristic of social interaction in SAD [44]. Additionally, together with SMN, VN has been suggested to show significantly greater BOLD responses in SAD for social threat in previous emotional fMRI study [45]. As for AN, regions within which have been found to related to dysfunction of cognitive reappraisal in SAD patients [46]. Consistent with these findings, our results imply a role for sensory networks in the perceptual and some other psychological impairments in SAD to variable extents.

Although the discriminating pattern above successfully allowed the identification of patients with SAD, the accuracy was not that high to achieve the goal of MVPA of automated MR image analysis in finding better sensitivity and specificity of antemortem diagnosis than what is currently possible [47]. While the performance, generalizability, and significance of the SVM findings would benefit from a large sample size and better feature selection methods [48], future studies incorporating large sample are needed to improve characterization of underlying features, as to establishment of a model which could most accurately predict new subjects for better classification. Furthermore, expanding feature selection to include other imaging properties, behavioral data, and genomic information may offer better discriminative information for predicting SAD.

In the exploratory analysis, no significant association was found between the test margin and clinical symptoms. In other words, the distance away from the hyperplane may not be driven or affected by the severity of symptoms as assessed by LSAS scores for a given subject, suggesting the discriminating pattern of ReHo obtained is relatively stable. This may be because the discrimination pattern produced derived mostly from the intergroup ReHo differences free from clinical ratings, which is of great significance since the identification of SAD will not be confounded by the psychological situations, reducing the rate of false negative findings resulting from individuals with mild symptoms.

It is noteworthy that there are some limitations implicated in the present study. First, although most of patients were drug-naïve, a small proportion of the SAD sample had taken medication before. However, we have prepared two weeks for the washing-out before scanning to reduce the confounding effect resulting from medication. Besides, given the effect of antianxiety medication in attenuating abnormally activated neural activity in social anxiety [49], we thus speculated that the medication effect would probably not exaggerate but instead tend to underestimate the capability of SVM in identifying patients. That might also be the reason why the present study did not find the abnormal alterations in amygdala due to attenuated amygdala responsiveness [50]. Second, as we have added whole voxels in the grey matter in the pattern analysis, the intrinsic structural differences may act as confounding factors in the pattern recognition analysis. However, ReHo and structural properties are different features in the SVM analysis; since we used the functional features implicated in ReHo, the confounding influence of structural differences was assumed marginal, if there were any. While we did not have sufficient structural images to conduct the same analysis to rule out confounding factors, future studies with different imaging modalities will be needed as a synthesized biomarker to strengthen the classification and achieve more reliable clinical diagnosis of this complex disorder. Finally, as a common psychiatric disorder, social anxiety has a potential correlation but differs from a personality trait known as shyness. While in current study, we only compare the cohort of SAD patients with healthy subjects, leaving an issue unresolved whether the application of SVM to ReHo would also discriminate SAD patients from mentally healthy people with shyness. The future studies may help to address this question by including a third group of subjects who have a level of shyness but without SAD.

\section{Conclusion}

This study used a MVPA method which is based on whole brain ReHo pattern, to distinguish individuals with SAD from healthy subjects. By presenting widespread differential map of coherence abnormalities which could be used to identify patients with SAD at the individual level, this study provides evidence that the ReHo of brain has the diagnostic potential and can possibly act as a supplementary approach to identify SAD, especially regions with high weight. Future studies with the integration of ReHo with other different imaging modality measurements may give a better insight into the imaging biomarkers of the condition.

\section{Conflict of Interests}

The authors declare that there is no conflict of interests regarding the publication of this paper.

\section{Authors' Contribution}

Wenjing Zhang and Xun Yang made equal contribution to this paper. 


\section{Acknowledgments}

This research was partially supported by the National Natural Science Foundation of China (Grant nos. 81222018, 81371527, 81030027, 81227002, and 81220108013), the Distinguished Young Scholars of Sichuan (Grant no. 2011JQ0005), the CMB Distinguished Professorship Award (no. F510000/G16916411), the National Key Technologies R\&D Program (Program no. 2012BAI01B03), the Ph.D. Programs Foundation of Ministry of Education of China (no. 20110181120033), and Program for Changjiang Scholars (PCSIRT, Grant no. IRT1272) of China. The authors thank all the subjects who participated in this study.

\section{References}

[1] M. B. Stein and D. J. Stein, "Social anxiety disorder," The Lancet, vol. 371, no. 9618, pp. 1115-1125, 2008.

[2] R. C. Kessler, T. C. Wai, O. Demler, and E. E. Walters, "Prevalence, severity, and comorbidity of 12-month DSM-IV disorders in the National Comorbidity Survey Replication," Archives of General Psychiatry, vol. 62, no. 6, pp. 617-627, 2005.

[3] A. A. Mather, M. B. Stein, and J. Sareen, "Social anxiety disorder and social fears in the Canadian military: prevalence, comorbidity, impairment, and treatment-seeking," Journal of Psychiatric Research, vol. 44, no. 14, pp. 887-893, 2010.

[4] M. C. Freitas-Ferrari, J. E. C. Hallak, C. Trzesniak et al., "Neuroimaging in social anxiety disorder: a systematic review of the literature," Progress in Neuro-Psychopharmacology and Biological Psychiatry, vol. 34, no. 4, pp. 565-580, 2010.

[5] A. Hahn, P. Stein, C. Windischberger et al., "Reduced restingstate functional connectivity between amygdala and orbitofrontal cortex in social anxiety disorder," NeuroImage, vol. 56, no. 3, pp. 881-889, 2011.

[6] W. Liao, H. Chen, Y. Feng et al., "Selective aberrant functional connectivity of resting state networks in social anxiety disorder," NeuroImage, vol. 52, no. 4, pp. 1549-1558, 2010.

[7] F. Liu, W. Guo, J.-P. Fouche et al., "Multivariate classification of social anxiety disorder using whole brain functional connectivity," Brain Structure and Function, pp. 1-15, 2013.

[8] F. Pereira, T. Mitchell, and M. Botvinick, "Machine learning classifiers and fMRI: a tutorial overview," NeuroImage, vol. 45, no. 1, pp. S199-S209, 2009.

[9] V. N. Vapnik, The Nature of Statistical Learning Theory, Statistics for Engineering and Information Science, Springer, New York, NY, USA, 2nd edition, 2000.

[10] G. Orrù, W. Pettersson-Yeo, A. F. Marquand, G. Sartori, and A. Mechelli, "Using Support Vector Machine to identify imaging biomarkers of neurological and psychiatric disease: a critical review," Neuroscience and Biobehavioral Reviews, vol. 36, no. 4, pp. 1140-1152, 2012.

[11] A. Frick, M. Gingnell, A. F. Marquand et al., "Classifying social anxiety disorder using multivoxel pattern analyses of brain function and structure," Behavioural Brain Research, vol. 259, pp. 330-335, 2014.

[12] X. Y. Long, X. N. Zuo, V. Kiviniemi et al., "Default mode network as revealed with multiple methods for resting-state functional MRI analysis," Journal of Neuroscience Methods, vol. 171, no. 2, pp. 349-355, 2008.
[13] Y. Zang, T. Jiang, Y. Lu, Y. He, and L. Tian, "Regional homogeneity approach to fMRI data analysis," NeuroImage, vol. 22, no. 1, pp. 394-400, 2004.

[14] T. Wu, X. Long, Y. Zang et al., "Regional homogeneity changes in patients with parkinson's disease," Human Brain Mapping, vol. 30, no. 5, pp. 1502-1510, 2009.

[15] Y. He, L. Wang, Y. Zang et al., "Regional coherence changes in the early stages of Alzheimer's disease: a combined structural and resting-state functional MRI study," NeuroImage, vol. 35, no. 2, pp. 488-500, 2007.

[16] J. Chen, Y. Xu, K. Zhang et al., "Comparative study of regional homogeneity in schizophrenia and major depressive disorder," American Journal of Medical Genetics B: Neuropsychiatric Genetics, vol. 162, no. 1, pp. 36-43, 2013.

[17] Y. Liu, K. Wang, C. YU et al., "Regional homogeneity, functional connectivity and imaging markers of Alzheimer's disease: a review of resting-state fMRI studies," Neuropsychologia, vol. 46, no. 6, pp. 1648-1656, 2008.

[18] J. J. Paakki, J. Rahko, X. Long et al., "Alterations in regional homogeneity of resting-state brain activity in autism spectrum disorders," Brain Research, vol. 1321, pp. 169-179, 2010.

[19] Q.-Z. Wu, D.-M. Li, W.-H. Kuang et al., "Abnormal regional spontaneous neural activity in treatment-refractory depression revealed by resting-state fMRI," Human Brain Mapping, vol. 32, no. 8, pp. 1290-1299, 2011.

[20] C.-Z. Zhu, Y.-F. Zang, Q.-J. Cao et al., "Fisher discriminative analysis of resting-state brain function for attention-deficit/ hyperactivity disorder," NeuroImage, vol. 40, no. 1, pp. 110-120, 2008.

[21] B. Schölkopf and A. J. Smola, Support Vector Machines and Kernel Algorithms, The Handbook of Brain Theory and Neural Networks, MIT Press, Cambridge, UK, 2002.

[22] T. Hastie, R. Tibshirani, J. Friedman, and J. Franklin, "The elements of statistical learning: data mining, inference and prediction," The Mathematical Intelligencer, vol. 27, no. 2, pp. 8385, 2005.

[23] S. Maji, A. C. Berg, and J. Maliks, "Classification using intersection kernel support vector machines is efficient," in Proceedings of the IEEE Conference on Computer Vision and Pattern Recognition (CVPR '08), pp. 1-8, Anchorage, Alaska, USA, June 2008.

[24] T. E. Nichols and A. P. Holmes, "Nonparametric permutation tests for functional neuroimaging: a primer with examples," Human Brain Mapping, vol. 15, no. 1, pp. 1-25, 2002.

[25] P. Golland and B. Fischl, "Permutation tests for classification: towards statistical significance in image-based studies," Information Processing in Medical Imaging, vol. 18, pp. 330-341, 2003.

[26] G. Sartori, S. Pellegrini, and A. Mechelli, "Forensic neurosciences: from basic research to applications and pitfalls," Current Opinion in Neurology, vol. 24, no. 4, pp. 371-377, 2011.

[27] C. Qiu, W. Liao, J. Ding et al., "Regional homogeneity changes in social anxiety disorder: a resting-state fMRI study," Psychiatry Research: Neuroimaging, vol. 194, no. 1, pp. 47-53, 2011.

[28] M. E. Raichle, A. M. MacLeod, A. Z. Snyder, W. J. Powers, D. A. Gusnard, and G. L. Shulman, "A default mode of brain function," Proceedings of the National Academy of Sciences of the United States of America, vol. 98, no. 2, pp. 676-682, 2001.

[29] R. L. Buckner, J. R. Andrews-Hanna, and D. L. Schacter, “The brain's default network," Annals of the New York Academy of Sciences, vol. 1124, no. 1, pp. 1-38, 2008.

[30] C. Gentili, E. Ricciardi, M. I. Gobbini et al., "Beyond amygdala: default Mode Network activity differs between patients with 
Social Phobia and healthy controls," Brain Research Bulletin, vol. 79, no. 6, pp. 409-413, 2009.

[31] J. M. Warwick, P. Carey, G. P. Jordaan, P. Dupont, and D. J. Stein, "Resting brain perfusion in social anxiety disorder: a voxel-wise whole brain comparison with healthy control subjects," Progress in Neuro-Psychopharmacology and Biological Psychiatry, vol. 32, no. 5, pp. 1251-1256, 2008.

[32] J. R. Simpson Jr., A. Z. Snyder, D. A. Gusnard, and M. E. Raichle, "Emotion-induced changes in human medial prefrontal cortex: I. During cognitive task performance," Proceedings of the National Academy of Sciences of the United States of America, vol. 98, no. 2, pp. 683-687, 2001.

[33] W. M. Kelley, C. N. Macrae, C. L. Wyland, S. Caglar, S. Inati, and T. F. Heatherton, "Finding the self? An event-related fMRI study," Journal of Cognitive Neuroscience, vol. 14, no. 5, pp. 785794, 2002.

[34] M. Corbetta and G. L. Shulman, "Control of goal-directed and stimulus-driven attention in the brain," Nature Reviews Neuroscience, vol. 3, no. 3, pp. 201-215, 2002.

[35] D. Mantini, M. G. Perrucci, C. del Gratta, G. L. Romani, and M. Corbetta, "Electrophysiological signatures of resting state networks in the human brain," Proceedings of the National Academy of Sciences of the United States of America, vol. 104, no. 32, pp. 13170-13175, 2007.

[36] A. D’Argembeau, F. Collette, M. Van Der Linden et al., "Selfreferential reflective activity and its relationship with rest: a PET study," NeuroImage, vol. 25, no. 2, pp. 616-624, 2005.

[37] D. M. Clark and F. McManus, "Information processing in social phobia," Biological Psychiatry, vol. 51, no. 1, pp. 92-100, 2002.

[38] S. G. Hofmann, "Cognitive mediation of treatment change in social phobia," Journal of Consulting and Clinical Psychology, vol. 72, no. 3, pp. 392-399, 2004.

[39] R. J. R. Blair, J. S. Morris, C. D. Frith, D. I. Perrett, and R. J. Dolan, "Dissociable neural responses to facial expressions of sadness and anger," Brain, vol. 122, no. 5, pp. 883-893, 1999.

[40] J. D. Greene, R. B. Sommerville, L. E. Nystrom, J. M. Darley, and J. D. Cohen, "An fMRI investigation of emotional engagement in moral judgment," Science, vol. 293, no. 5537, pp. 2105-2108, 2001.

[41] M. R. Milad and S. L. Rauch, "The role of the orbitofrontal cortex in anxiety disorders," Annals of the New York Academy of Sciences, vol. 1121, pp. 546-561, 2007.

[42] M. Tillfors, T. Furmark, I. Marteinsdottir et al., "Cerebral blood flow in subjects with social phobia during stressful speaking tasks: a PET study," The American Journal of Psychiatry, vol. 158, no. 8, pp. 1220-1226, 2001.

[43] J. R. Simpson Jr., W. C. Drevets, A. Z. Snyder, D. A. Gusnard, and M. E. Raichle, "Emotion-induced changes in human medial prefrontal cortex: II. During anticipatory anxiety," Proceedings of the National Academy of Sciences of the United States of America, vol. 98, no. 2, pp. 688-693, 2001.

[44] S. M. Bögels and W. Mansell, "Attention processes in the maintenance and treatment of social phobia: hypervigilance, avoidance and self-focused attention," Clinical Psychology Review, vol. 24, no. 7, pp. 827-856, 2004.

[45] P. R. Goldin, T. Manber, S. Hakimi, T. Canli, and J. J. Gross, "Neural bases of social anxiety disorder: emotional reactivity and cognitive regulation during social and physical threat," Archives of General Psychiatry, vol. 66, no. 2, pp. 170-180, 2009.

[46] M. Ziv, P. R. Goldin, H. Jazaieri, K. S. Hahn, and J. J. Gross, "Emotion regulation in social anxiety disorder: behavioral and neural responses to three socio-emotional tasks," Biology of Mood \& Anxiety Disorders, vol. 3, article 20, 2013.

[47] S. Klöppel, C. M. Stonnington, C. Chu et al., "Automatic classification of MR scans in Alzheimer's disease," Brain, vol. 131, no. 3, pp. 681-689, 2008.

[48] H. Ung, J. E. Brown, K. A. Johnson, J. Younger, J. Hush, and S. Mackey, "Multivariate classification of structural MRI data detects chronic low back pain," Cerebral Cortex, vol. 24, no. 4, pp. 1037-1044, 2014.

[49] T. Furmark, L. Appel, Å. Michelgård et al., "Cerebral blood flow changes after treatment of social phobia with the neurokinin-1 antagonist GR205171, citalopram, or placebo," Biological Psychiatry, vol. 58, no. 2, pp. 132-142, 2005.

[50] V. Faria, L. Appel, F. Åhs et al., "Amygdala subregions tied to SSRI and placebo response in patients with social anxiety disorder," Neuropsychopharmacology, vol. 37, no. 10, pp. 2222$2232,2012$. 

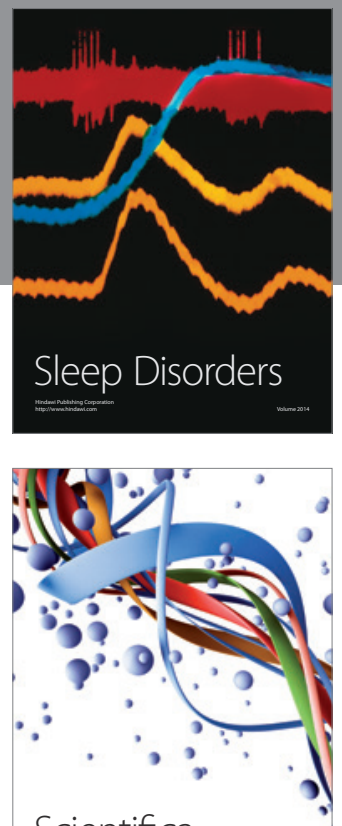

Scientifica
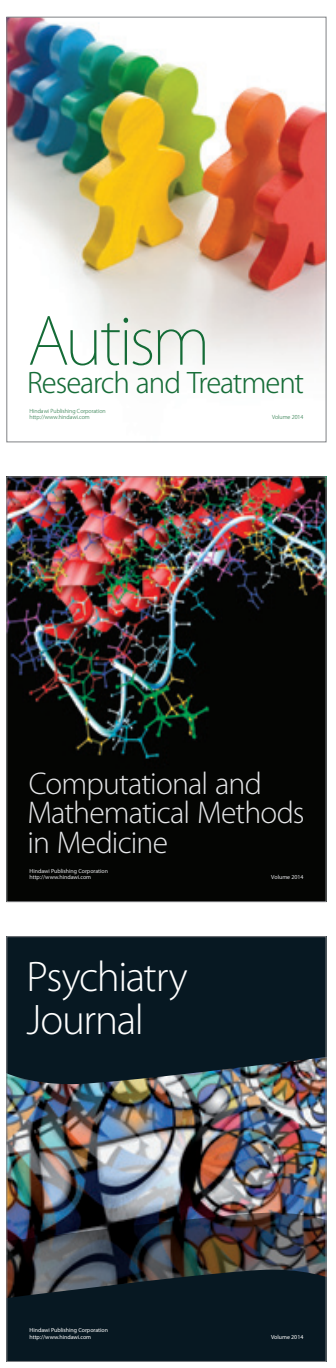
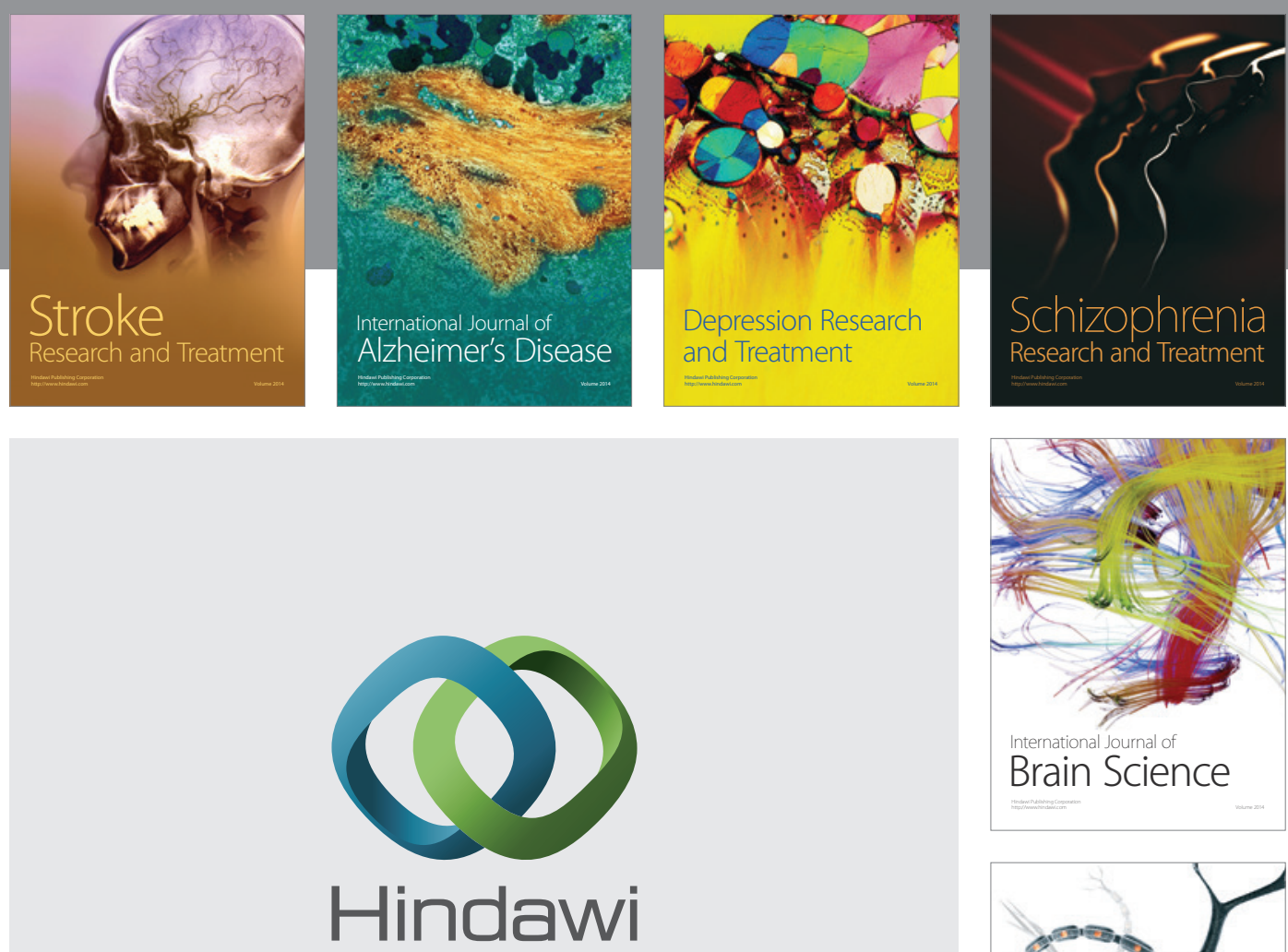

Submit your manuscripts at

http://www.hindawi.com
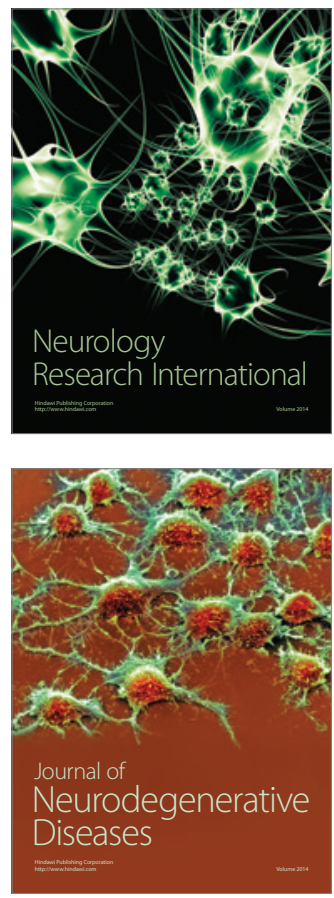

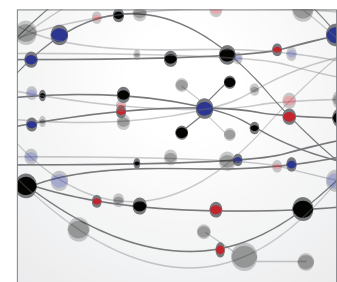

The Scientific World Journal
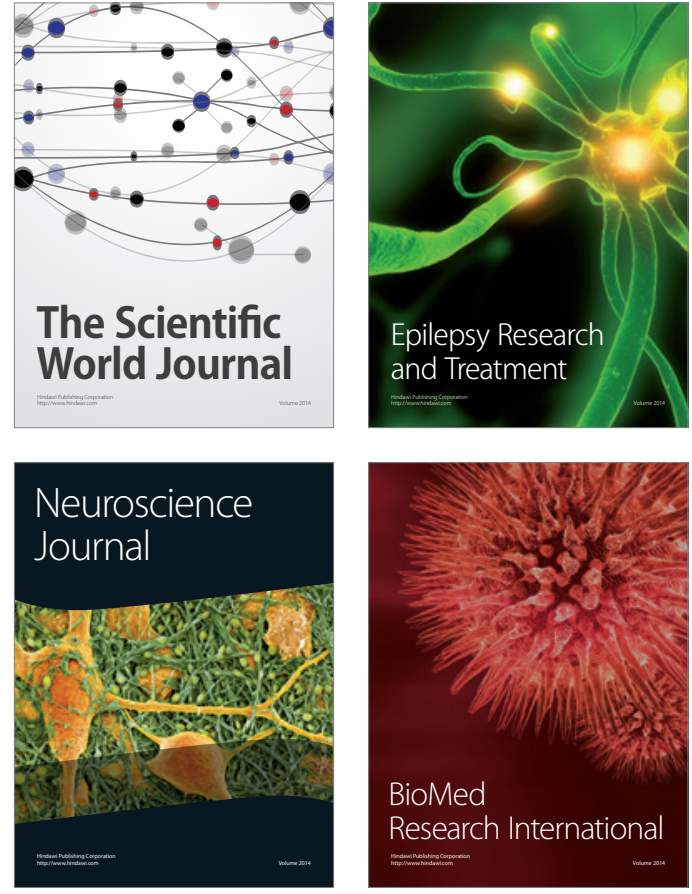

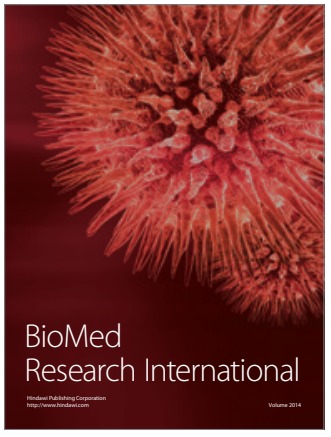

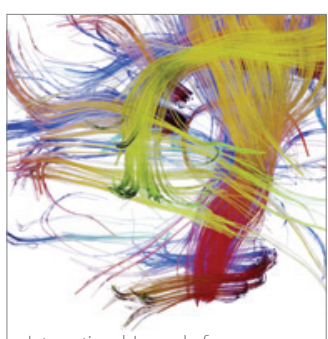

Brain Science

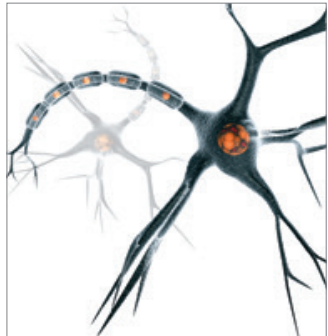

Neural Plasticity
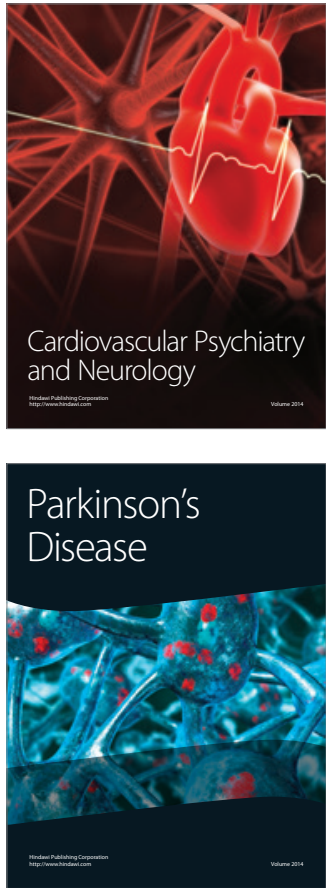